\title{
Construction of Visual Inspection Database for Catenary on High-speed Railways
}

\author{
Yan-guo Wang ${ }^{1}$, Dapeng $\mathrm{Xie}^{1}$, Haiying Wang ${ }^{1}$, Xiangdong $\mathrm{Li}^{2}$, Jing Shi ${ }^{1}$, Hailang $\mathrm{Li}^{1}$, Kexin Xiao ${ }^{2}$ and Jincai $\mathrm{Li}^{2}$ \\ ${ }^{1}$ Institute of Infrastructure Inspection, China Academy of Railway Sciences, Beijing 100081, China \\ ${ }^{2}$ Beijing IMAP Technology Co., Ltd, Beijing 100081, China
}

\begin{abstract}
With the rapid development of computer vision, techniques of machine vision and visual inspection have been applied into the inspection of catenary on high-speed railways. Visual inspection systems have been developed and super-high-resolution images are captured to check the status of catenary components. Automatic recognition of defects becomes very important since the number of images is too huge to be manually checked one by one. However, it is not easy for the development of recognition algorithms on catenary components. There are many types of defects to be checked on different kinds of catenary components, but the number of defect images is too small in real world. In this paper, a solution was proposed and implemented. An on-site data acquisition system was designed and developed, and different types of defects were manually made on different catenary components beforehand. Finally, a visual inspection database was successfully constructed, including plenty of different kinds of catenary components, different types of defects, in different inspection conditions. The visual inspection database will be of great use in the development and test of recognition algorithms for catenary.
\end{abstract}

\section{Introduction}

Catenary [1] is the one of the main infrastructures along high-speed railways which supplies power for the trains. Regular inspections have to be performed in order to guarantee the status of catenary. With the rapid development of computer vision [2,3], techniques of machine vision and visual inspection have been applied into the inspection of catenary [4, 5]. Several visual inspection systems have been developed and super-highresolution images are captured to check the status of catenary components [6-8].

However, the subsequent analysis on the visual inspection data is quite a difficult task. The number of images is too huge so that it will be very inefficient to be manually checked one by one. Automatic analysis is necessary but the development of the detection algorithms is not easy. There are so many kinds of catenary components to be checked, and hundreds of defect types to be automatically detected on different components. Development and test of recognition algorithms rely on a visual inspection database with image data of high quality, which should cover different catenary components and different types of defects. Unfortunately, defects on catenary components are rarely seen in real world and difficult to be collected, so that the number of defect images is too small for the development and test of recognition algorithms.

In this paper, a solution was proposed to construct the visual inspection database. The data acquisition was performed on a typical railway line which was under construction. An on-site data acquisition system was designed and developed, and different types of defects were manually made on different catenary components beforehand. Finally, a visual inspection database was successfully constructed, including plenty of different kinds of catenary components, different types of defects, in different inspection conditions.

The rest of the paper is organized as follows. The onsite data acquisition system is described in section 2 . The arrangement of defects per-made and data acquisition is discussed in section 3. The final visual inspection database is reported in section 4 . Then we draw the conclusions.

\section{Data acquisition system}

Catenary is composed of contact wire, cantilever, additional wire, and hanging post (in tunnels), which is illustrated in Fig. 1. There are many kinds of components in different regions of catenary, which vary greatly in size and location. 


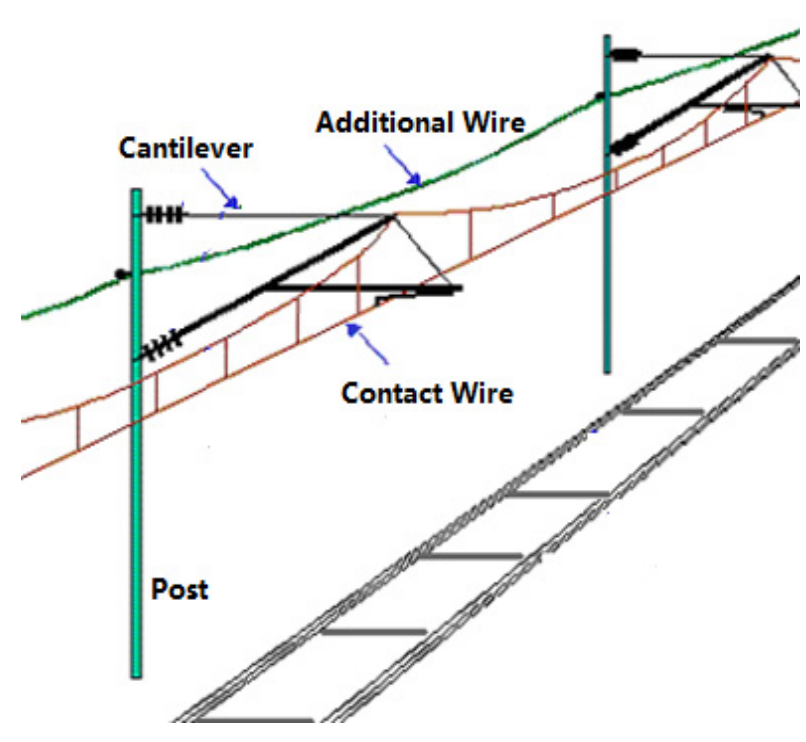

Figure 1. Structure of catenary

The data acquisition system was designed based on catenary maintenance ladders (Fig. 2), which can be operated on railway lines under construction.

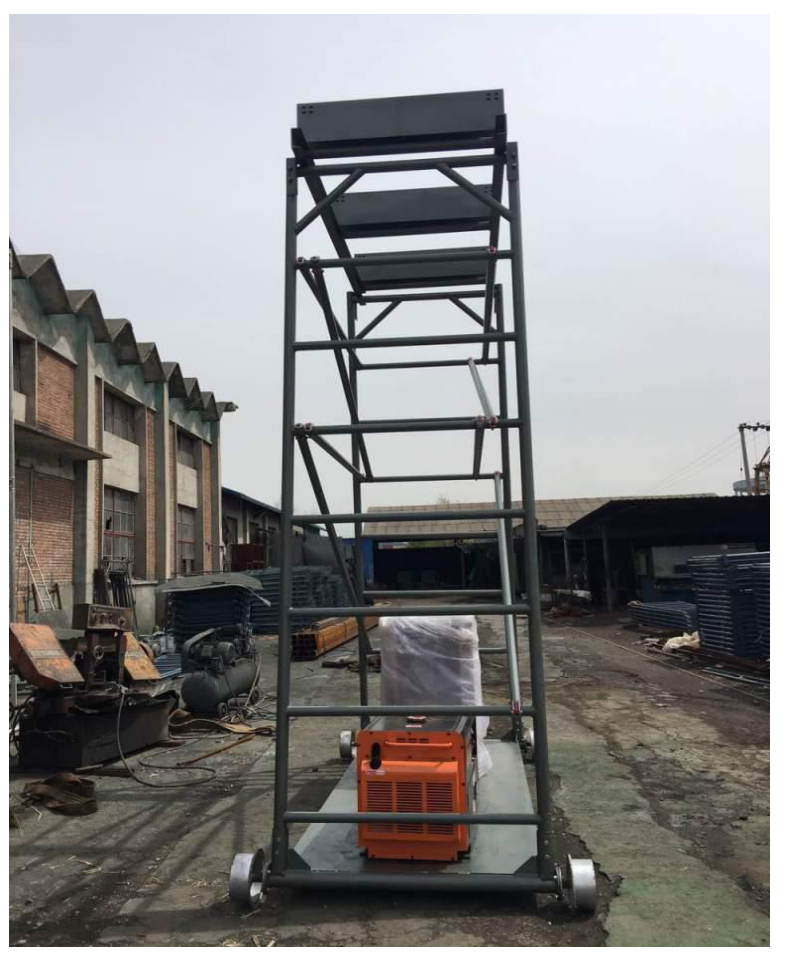

Figure 2. Catenary maintenance ladder.

18 super-high-resolution cameras are used to capture detailed images of catenary components in different view directions, with an image resolution up to 29 megapixels. Fig. 3 illustrates the arrangement of the camera system.

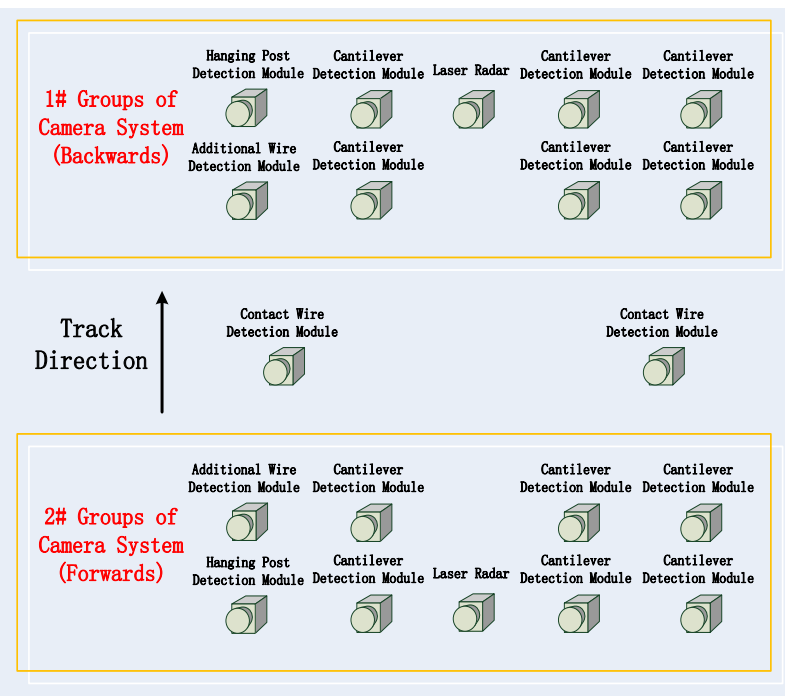

Figure 3. Camera system arrangement.

Most catenary components concentrate at catenary posts, and we have to capture images at a proper distance to each post. Two laser radars are utilized here to give an accurate trigger signal for the camera system. The laser radars continuously measure the distance from the camera system to next catenary post, and a trigger signal will be generated and distributed to the camera system, when the distance reaches a pre-define value.

The final design of the camera system is showed in Fig. 4.

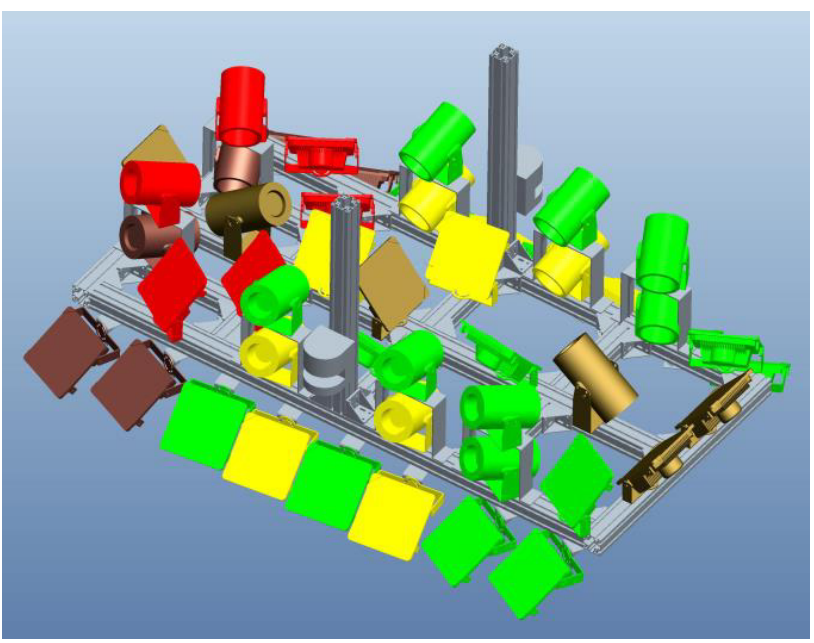

Figure 4. Camera system design.

Table 1 gives the specifications of each detection module in the camera system.

Table 1. Specifications of camera system.

\begin{tabular}{|c|c|c|c|}
\hline $\begin{array}{c}\text { Detection } \\
\text { Modules }\end{array}$ & $\begin{array}{c}\text { Camera } \\
\text { Resolution } \\
\text { (megapixel) }\end{array}$ & $\begin{array}{c}\text { Number } \\
\text { of } \\
\text { Cameras }\end{array}$ & View Directions \\
\hline Cantilever & 29 & 12 & $\begin{array}{c}\text { Forwards \& } \\
\text { backwards }\end{array}$ \\
\hline $\begin{array}{c}\text { Contact } \\
\text { Wire }\end{array}$ & 25 & 2 & Left \& right \\
\hline
\end{tabular}




\begin{tabular}{|c|c|c|c|}
\hline $\begin{array}{c}\text { Additional } \\
\text { Wire }\end{array}$ & 16 & 2 & $\begin{array}{c}\text { Forwards \& } \\
\text { backwards }\end{array}$ \\
\hline $\begin{array}{c}\text { Hanging } \\
\text { Post }\end{array}$ & 16 & 2 & $\begin{array}{c}\text { Forwards \& } \\
\text { backwards }\end{array}$ \\
\hline Laser Radar & $/$ & 2 & $\begin{array}{c}\text { Forwards \& } \\
\text { backwards }\end{array}$ \\
\hline
\end{tabular}

The arrangement of the machine cabinet is illustrated in Fig. 5. There are camera control unit and light control unit, which control the power supply and trigger signal of cameras and lighting device respectively. Trigger and control unit is in charge of the signal processing of laser radars, also the generation and distribution of trigger signals. The final image data acquisition and storing is managed by a data acquisition server. There is also an UPS here in order to guarantee the stable power supply of the system.

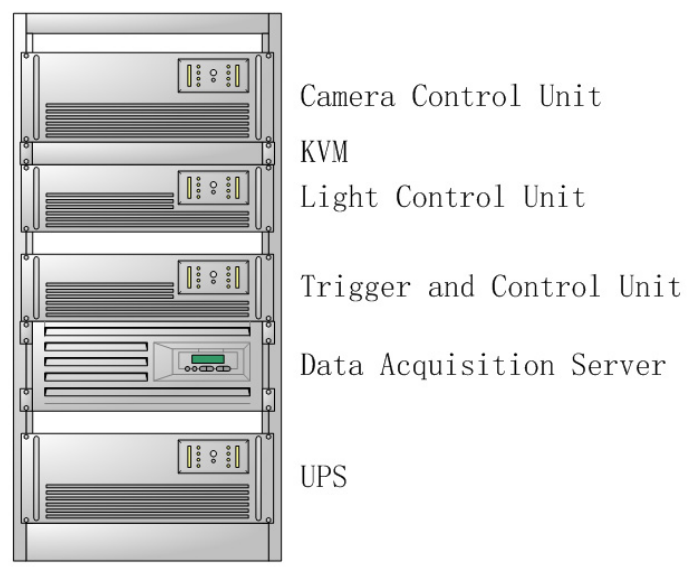

Figure 5. Machine cabinet.

The graphic user interface of the acquisition system is showed in Fig. 6.

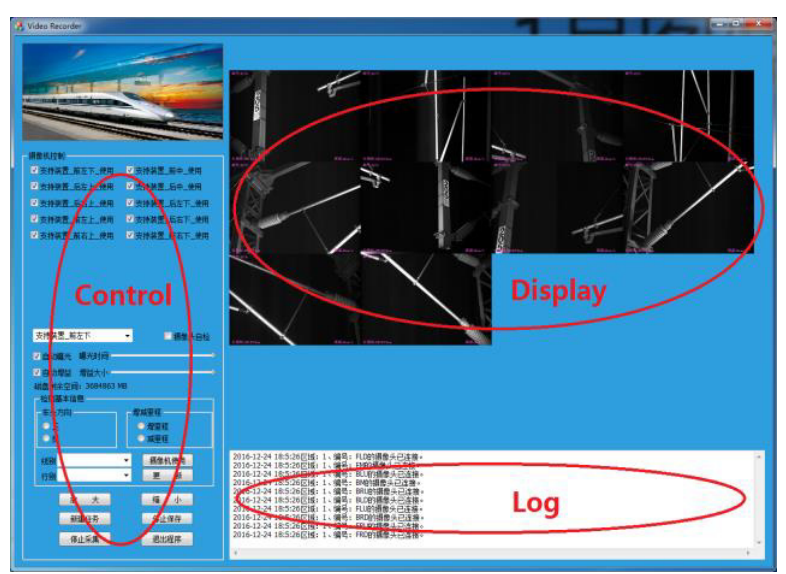

Figure 6. Graphical user interface.

\section{Defects pre-made and data acquisition}

The data acquisition was performed on some selected sections of a typical high-speed railway which was under construction. Straight lines, curves, stations, tunnels were all involved, and the acquisition was performed both in day and at night, in order to ensure the representativeness and completeness of the database.

Hundreds of different types of defects had been manually made on different kinds of catenary components beforehand. Super-high-resolution image data were captured both with and without the defects, also both in day and at night. The pre-made defects were restored after the acquisition, so as to guarantee the safety and quality of catenary.

Fig. 7 shows the process of manually-making defects, and the data acquisition is showed in Fig. 8.

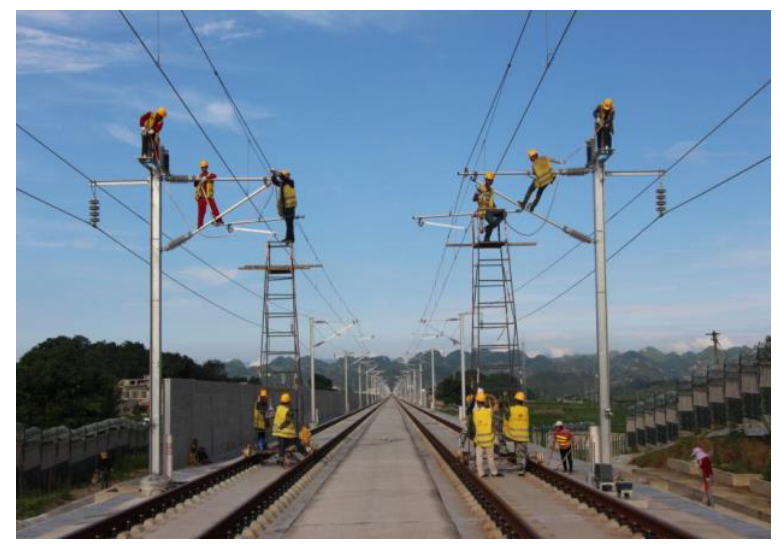

Figure 7. Defects pre-made on catenary.

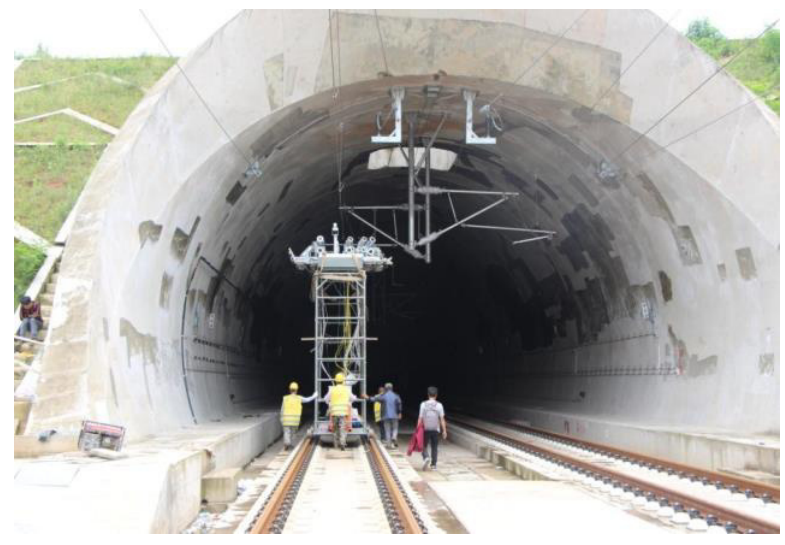

Figure 8. Data acquisition.

\section{Visual inspection database}

The final visual inspection database is summarized in Table 2. It covers different kinds of catenary components, and plenty of different types of defects data, in different inspection conditions.

Table 2. Visual inspection database.

\begin{tabular}{|c|c|c|c|}
\hline Types & $\begin{array}{c}\text { Number of } \\
\text { posts }\end{array}$ & Mileage (km) & $\begin{array}{c}\text { Number of } \\
\text { images }\end{array}$ \\
\hline Normal data & 1665 & 83.2 & 19980 \\
\hline Defects data & 87 & 4.4 & 1044 \\
\hline Total & 1752 & 87.6 & 21024 \\
\hline
\end{tabular}


The data acquisition was performed both in day and at night, which is summarized in Table 3.

Table 3. Visual inspection database.

\begin{tabular}{|c|c|c|c|}
\hline $\begin{array}{c}\text { Inspection } \\
\text { conditions }\end{array}$ & $\begin{array}{c}\text { Number of } \\
\text { posts }\end{array}$ & $\begin{array}{c}\text { Mileage } \\
(\mathbf{k m})\end{array}$ & $\begin{array}{c}\text { Number of } \\
\text { images }\end{array}$ \\
\hline In day & 364 & 20.0 & 4368 \\
\hline At night & 1388 & 67.6 & 16656 \\
\hline Total & 1752 & 87.6 & 21024 \\
\hline
\end{tabular}

Some enlarged normal data are showed in Fig. 9 11. Some defects data are showed in Fig. 12 17, with the position of defects labelled using red ellipses.

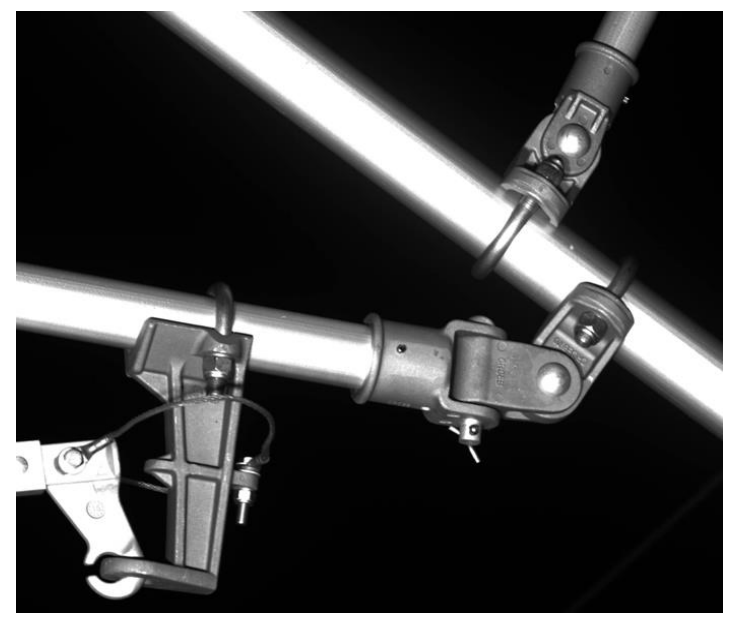

Figure 9. Normal data (enlarged).

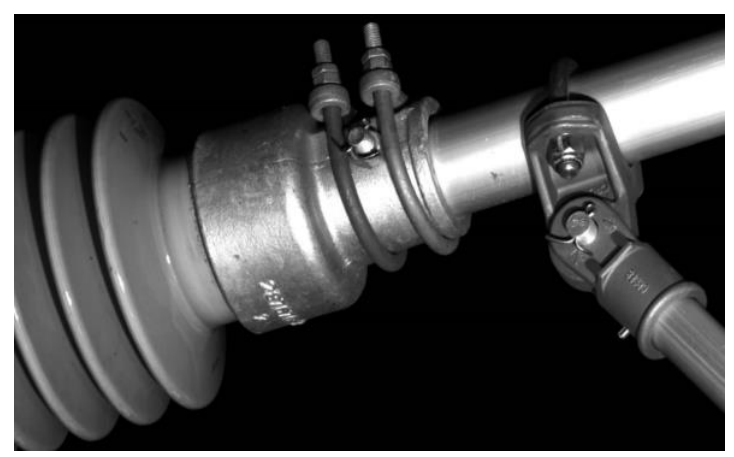

Figure 10. Normal data (enlarged).

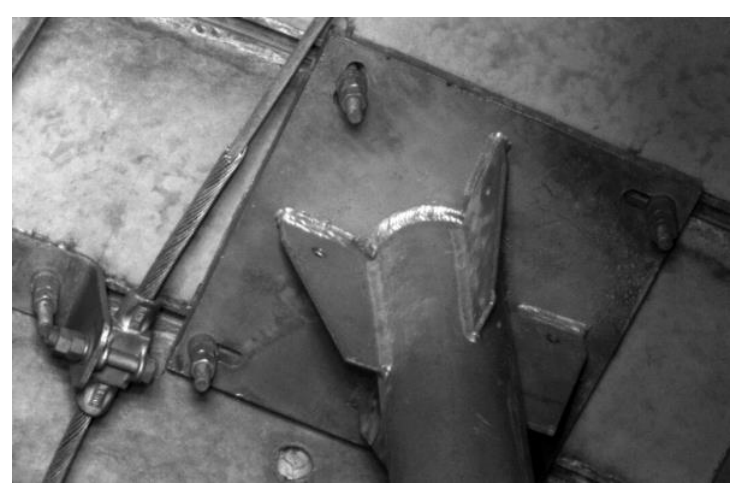

Figure 11. Normal data (enlarged).

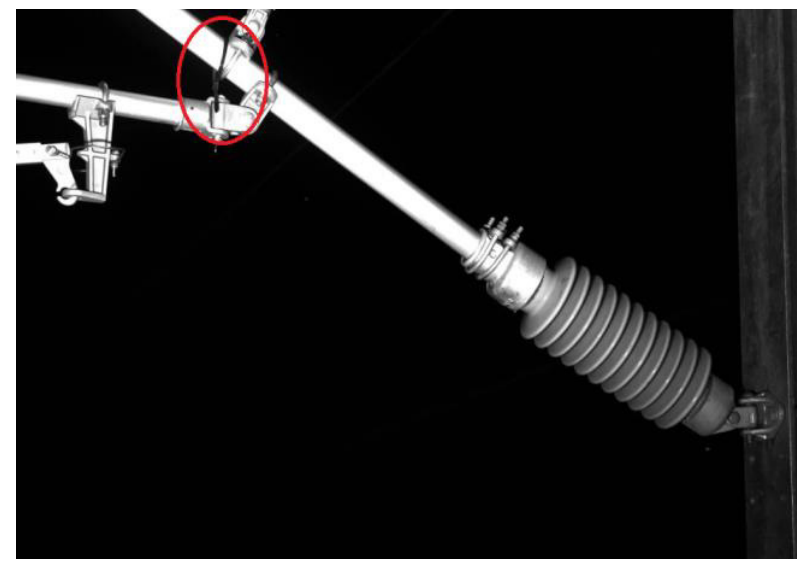

Figure 12. Data with pre-made defects.

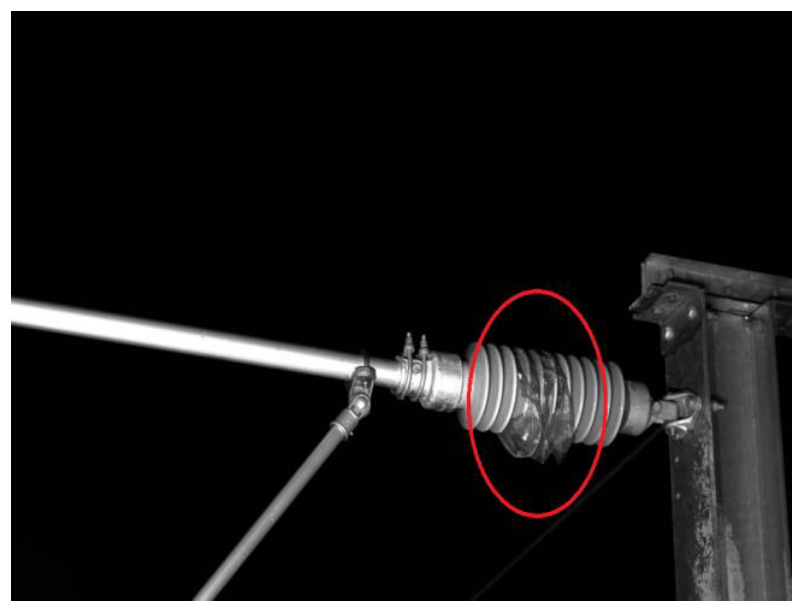

Figure 13. Data with pre-made defects.

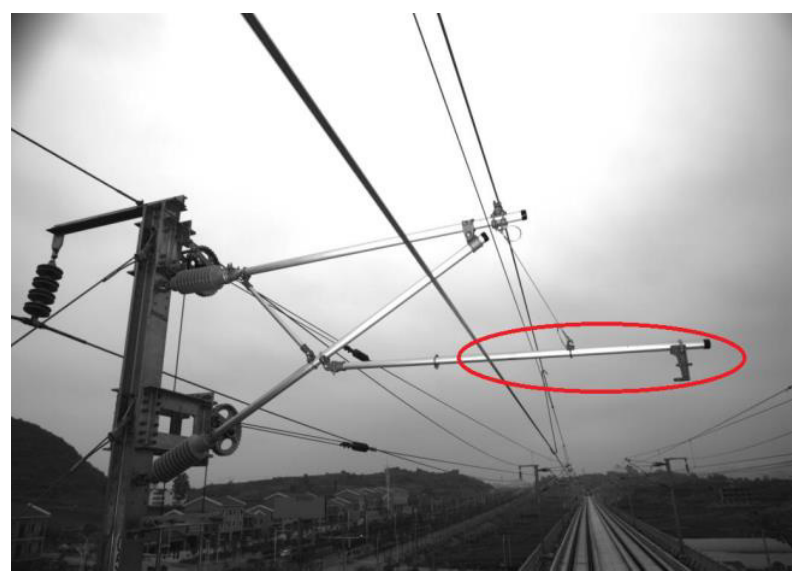

Figure 14. Data with pre-made defects. 


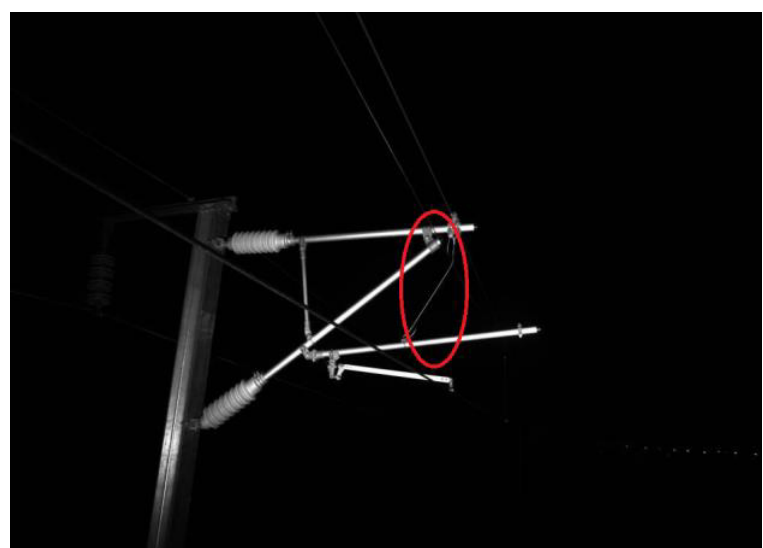

Figure 15. Data with pre-made defects.

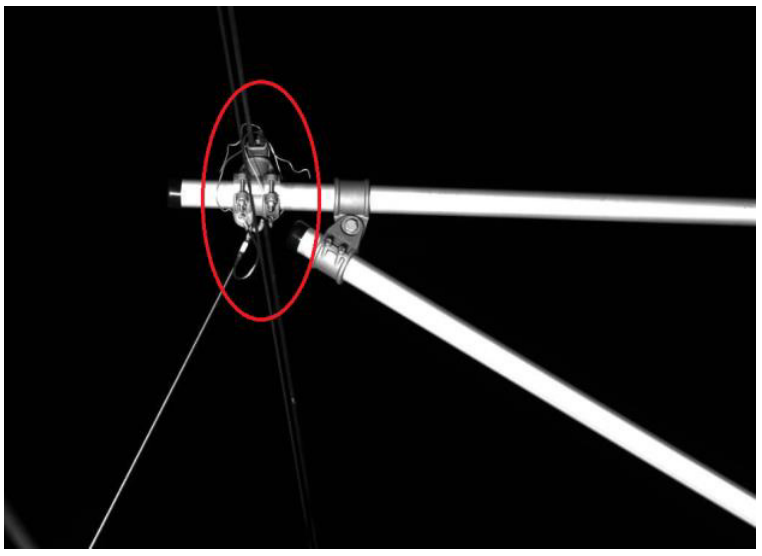

Figure 16. Data with pre-made defects.

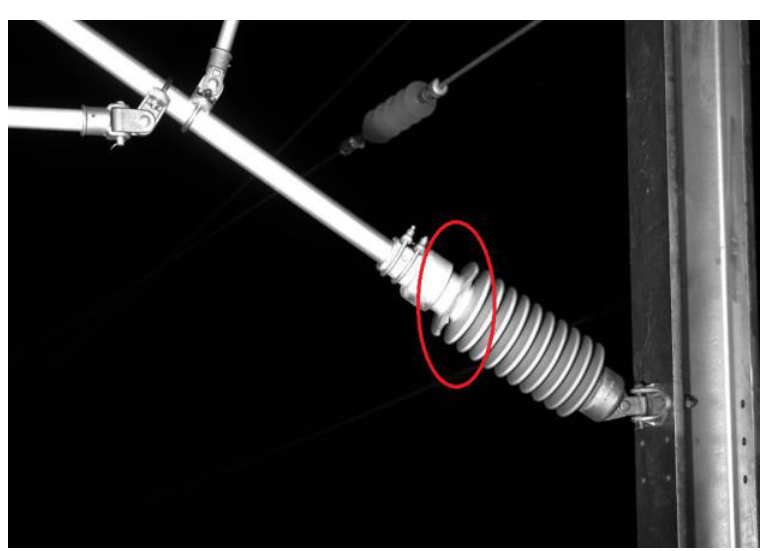

Figure 17. Data with pre-made defects.

\section{Summary}

Technologies of computer vision and visual inspection have been applied into the inspection of catenary on highspeed railways. However, the automatic analysis of visual inspection data relies on a high quality visual inspection database, which is very difficult to be collected in the conventional manner. In this paper, an on-site data acquisition system was proposed, and plenty of image data are captured with different types of defects pre-made on different kinds of catenary components. The final visual inspection database will be of great use in the future development and test of recognition algorithms for catenary.

\section{Acknowledgement}

This research was partly supported by the National Key Basic Research Program of China (973 Program) (Grant No. 2013CB329406), China Railway Corporation Program on Scientific Research and Development Project (Grant No. 2015T003-A), and Beijing IMAP Technology Co., Ltd Program on Scientific Research and Development Project (Grant No. 2016JJXM11).

\section{References}

1. F. Kiessling, R. Puschmann, A. Schmieder, et al. "Contact lines for electric railways (second edition)." Publicis Kommunikations Agentur GmbH, Germany (2009).

2. D. A. Forsyth and J. Ponce. "Computer vision: a modern approach." Prentice Hall, America (2003).

3. E. R. Davies. "Machine vision: theory, algorithms, practicalities (third edition)." Elsevier, Singapore (2005).

4. Yan-guo Wang, Zhongguo Sun, Shiping Gu, et al. "Real-time measurement for the angle of steady arm on high-speed railways." Proc. of the 10th World Congress of Railway Research (2013).

5. Wei Zhou, Zhongguo Sun, Shengwei Ren, et al. "Measurement method for geometric parameters of overhead contact line based on multi-view stereovision." China Railway Science, 36(5): (In Chinese). (2015) 104-109.

6. U. Richter and R. Schneider. "Automatic optical inspection for catenary." Railway Engineer (Chinese Edition), 7(1), (2001) 24-28.

7. U. Richter. "Contact and non-contact comprehensive catenary inspection system." Railway Technology Review, (2009) 8-10.

8. Yan-guo Wang, Wei Zhou, Dapeng Xie, et al. "Automatic Visual Inspection for Catenary on Highspeed Railways." Proc. of the First International Conference on Rail Transportation (2017). 